\title{
LA TÉCNICA PAOO UNA ALTERNATIVA EN EL TRATAMIENTO ORTODONTICO: REVISIÓN DE LITERATURA
}

\section{TECHNICAL PAOO AN ALTERNATIVE IN THE ORTHODONTIC TREATMENT: REVISION OF LITERATURE}

\author{
Mercado M. Sively. ${ }^{1 *}$ Mercado P. Jorge ${ }^{2}$ Mamani C. Luz D ${ }^{3}$ Tapia C. Rildo $^{4}$ Heredia-Veloz David. $^{5}$ \\ ${ }^{1}$ Doctor en Salud Pública, Especialista en Ortodoncia Ortopedia Maxilar, Docente de la Universidad Andina Néstor Cáceres \\ Velásquez.Perú. \\ ${ }^{2}$ Doctor en Salud Pública, Especialista en Periodoncia e Implantología Oral , Docente Principal de la Universidad Nacional \\ del Altiplano.Perú. \\ ${ }^{3}$ Doctora en Salud Pública, Especialista en Rehabilitación Oral, Docente de la Universidad Nacional del Altiplano.Perú. \\ ${ }^{4}$ Doctor en Estomatolgía, Especialista en Endodoncia, Docente de la Universidad Andina Néstor Cáceres Velásquez.Perú. \\ ${ }^{5}$ Odontólogo Universidad de Cuenca.Ecuador.
}

*goty20@hotmail.com

\begin{abstract}
Resumen
Una alternativa que ha brindado beneficios en el tratamiento ortodóntico al disminuir la reabsorción radicular y brindar mejor estabilidad después del tratamiento es la terapia ortodoncica periodontalmente avanzada, para conseguir éxito en el tratamiento debe haber una estricta cooperación entre el ortondocista y periodoncista durante la planificación del caso. En esta terapia es necesario realizar un abordaje quirúrgico llamado corticotomia que consiste en acceder a la cortical óseo, este proceso activa una cascada fisiológica encargada de producir el movimiento ortodontico acelerado. Existen resultados clínicos e histológicos prometedores, comprobados por la evidencia científica. PAOO aumenta el movimiento ortodóntico después de la decorticacion alveolar y el aumento óseo contribuye a la unión ósea brindando estabilidad del tratamiento al largo plazo; aumentar el volumen alveolar y reforzar el periodonto.
\end{abstract}

Palabras clave: ortodoncia, matriz osea, movimiento dentario, aceleración.

\begin{abstract}
An alternative that has provided benefits in orthodontic treatment to reduce root resorption and provide better stability after treatment is periodontally advanced orthodontic therapy, to achieve success in treatment there must be strict cooperation between the orthodontist and periodontist during the planning of the case. In this therapy it is necessary to perform a surgical approach called corticotomy consisting of access to the cortical bone, this process activates a physiological cascade responsible for producing accelerated orthodontic movement. There are promising clinical and histological results, as evidenced by scientific evidence. PAOO increases orthodontic movement after alveolar decortication and bone augmentation contributes to bone union providing long-term treatment stability; increase the alveolar volume and strengthen the periodontium.
\end{abstract}

Key words: orthodontics, bone matrix, tooth movement, acceleration.

\section{INTRODUCCIÓN}

Existe evidencia científica de métodos y técnicas para acelerar el movimiento ortodóncico. Una de las técnicas es la corticotomía, también llamada decorticación alveolar selectiva (SAD), Frost describió esta teoría y manifestó que el recambio óseo producto de una respuesta fisiológica al fe- nómeno de aceleración regional. ${ }^{1,2}$ Henrick kole en 1959 fue el primero en describir una técnica quirúrgica para conseguir movimientos ortodonticos mediante corticotomía, ya que sé que consideraba que corticales ocasionaban impedimento en el movimiento dentario y se creía que al cambiarlas morfológicamente el diente se movería en menor tiempo. Kole en su técnica quirúrgica realizó un colgajo de espesor 
total y realizó cortocotomías en sitios interradiculares, al ser una técnica muy invasiva fue poco aceptada. ${ }^{3,4}$ Esta nueva posibilidad enel tratamiento de ortodoncia ha despertado el interés en los pacientes, especialmente en los adultos quienes se negaban a recibir tratamiento ortodóntico debido que los resultados se manifestaban luego de periodos largos. El tratamiento ortodóntico en pacientes adultos es rechazado por el tiempo prolongado en la obtención de resultados ,poniendo en juego la salud oral del paciente, por ello ha surgido como alternativa de un tratamiento ortodóntico en menor tiempo la cortocotomia, que consiste en realizar cortes en la cortical vestibular, palatina o lingual para estimular el fenómeno regional acelerado (RAP), disminuyendo el tiempo del tratamiento ortodóntico de 3 a 4 veces produciendo menos efectos adversos como reabsorción radicular y poca estabilidad después del mismo. ${ }^{5-7}$ Wilcko et al. Observó que la ortodoncia rápida (PAOO) ofrece un tratamiento de 6 a 8 meses. Ellos cuestionaron el mandato de Köle "el bloque óseo" y ofreció una hipótesis alternativa que el movimiento del diente rápido era el resultado del marcado pero transeúnte mecanismo de recalcificación y de descalcificación del alveolo. Hiele, cirujano ortopédico, había descrito una correlación directa entre el grado y proximidad de trauma del hueso e intensidad de contestación curativa fisiológica que él acuñó el Fenómeno de Aceleración Regional (RAP), o GOLPE SECO (Escarcha 1983), y el mecanismo de descalcificación-recalcificación descrito por Wilcko et al. $(2001,2003)$ era consistente con el GOLPE SECO. ${ }^{8}$ En 1959, Köle $^{9}$ informó que combinando la ortodoncia con la cirugía (corticotomia) se completa el movimiento dentario activo en los casos ortodónticos adultos en 6 a 12 semanas. Los cortes de corticotomia interproximal estaban extendidos a través del espesor entero de la capa cortical, sólo escasamente penetrante en el hueso medular. Esta revisión bibliográfica tiene como objetivo de describir los fundamentos biológicos, las aplicaciones clínicas de la ortodoncia facilitada por corticotomía, con la técnica descrita por Wilcko (PAOO).

\section{LA RAZÓN BIOLÓGICA- FENÓMENO REGIO- NAL ACELERATORIO}

La técnica clínica que involucra la decorticacion alveolar selectiva, junto a la técnica PAOO es una forma de diseñar el tejido periodontal para producir una osteopenia transeúnte y la producción alta y adyacente al sitio de la lesión (osteopenia-osteoformacion).

Se ha definido al movimiento ortodóntico como una respuesta biológica ocasionada por la aplicación de fuerzas externas. La velocidad de este movimiento ortodóntico depende de las características físicas de la fuerza aplicada el tamaño y respuesta biológica del ligamento periodontal. ${ }^{10,11}$ Estas tensiones alteran la vascularización y el flujo de sangre del ligamento periodontal liberando neurotransmisores, citocinas, factores de crecimiento, y metabolitos del ácido araquidónico que producen repuestas de varios tipos de células alrededor del diente permitiendo la aposición y resorción de los tejidos. ${ }^{12}$ Al realizar una corticotomia, la cicatrización mejora debido al RAP. El RAP (Fenómeno de Aceleración Regional) es una respuesta local y transitoria de remodelación seguida de la cicatrización postquirúrgica de la cortical ósea frente a un estímulo nocivo ${ }^{13}$ en donde la formación tisular es de 2 a 10 veces más rápida que los procesos normales regenerativos. Cada vez que se crea un daño mecánico en el periodonto creado por una fuerza ortodóntica, las plaquetas migran de los vasos sanguíneos al espacio extravascular. Estas plaquetas son una fuente importante (grow factor- factor de crecimiento). ${ }^{14}$ Frost ${ }^{15}$ mencionó que después de pocos días de producida la lesión ósea comienza el RAP. Llegando a sumaxima actividad uno o dos meses, pero este proceso podría terminar hasta dentro de veinte y cuatro meses. El RAP se prolonga; cuando el RAP disminuye el estado de osteopenia desaparece. Al terminar los movimientos ortodónticos se crea un ambiente favorable para la remineralización alveolar. Lee et al, ${ }^{16,17}$ por medio de microtomografía computarizada encontraron que la osteotomía producía cambios semejantes a la de un sitio de distracción distal, mientras que la corticotomía produce pérdida ósea regional, típica del RAP. Estas diferencias se producen debido al incremento de la movilidad del segmento osteotomizado, donde la cicatrización es similar a la de una fractura, mientras que en los sitios de corticotomía, la cicatrización se llevó a cabo por medio de aperturas hacia los espacios vasculares medulares adyacentes, exponiendo el sitio quirúrgico a un aumento en el potencial de cicatrización, manteniendo al segmento involucrado en un estado estable. Wang et al ${ }^{18}$ demostró los resultados detallados mediante un estudio histológico con inmunohistotinción, en este se observaron tres fases de cicatrización ósea: fase de resorción (a los 3 días) caracterizada por la pérdida ósea en las raíces y mayor número de osteoclastos, la segunda fase es de reemplazo (a los 21 días), caracterizándose por la sustitución de tejido multicelular por el tejido ósea encontrando la presencia de células mesenquimales similares a los osteoblastos. Por último, se produce la fase de mineralización (a los 60 días) determinado por la formación de hueso primario. El recambio óseo es acelerado después de un daño en el hueso como una fractura, una osteotomía o agregado de injerto óseo. ${ }^{19}$ En el RAP el número de osteoblastos y osteoclastos aumenta por los mecanismos locales de mediadores multicelulares que contienen células precursoras, células de soporte, capilares sanguíneos y linfa. La activación con corticotomías produce una activación ósea, provocada por un proceso de reabsorción y formación en el periodonto, al cabo de 3 semanas de la decorticacion el número de osteoclastos y proceso de aposicion son mayores que a los resultados realizados mediante un tratamiento de ortodoncia convencional. Además de esta situación se distingue en la zona de la injuria que la cantidad de hueso esponjoso calcificado disminuye, aumentando la superficie del ligamento periodontal dos veces más rápido que lo normal. ${ }^{20,21}$ 
Hay tres principios en los tejidos asociados, en el tratamiento de corticotomías. El primer principio se produce cuando la decorticación inicia un proceso de reparación y de producción de células progenitoras y de agentes osteoinductores. El segundo principio se produce cuando en el recambio óseo se origina una disminución de la densidad ósea, pero no del volumen. El tercer y último principio es obtenido cuando este recambio óseo se produce en un lugar anatómico adecuado, adyacente al lugar donde se realizó la corticotomía. ${ }^{22}$ Los tejidos que se forman alrededor del alveolo, en el área en el que se desea mover un diente, responde a las fuerzas biomecánicas y al movimiento dental rápido. La osteopenia del hueso medular es alta en la zona más cercana a la corticotomía. ${ }^{22}$ Clínicamente se produce menor reabsorción en las raíces en la técnica ortodoncica acelerada con corticotomías, que en la ortodoncia convencional. Esto debido a los procesos fisiológicos producidos después de una cirugía la misma que ocasiona una rápida reacción del hueso alveolar en las cavidades de la médula ósea, llevando una menor hialinización del PDL en la pared alveolar. ${ }^{23,24} \mathrm{Un}$ tratamiento ortodóncico de corto tiempo, es ventajosos o para los tejidos periodontales, lo cual reduce substancialmente el tiempo disponible para que los biofilms bacterianos relativamente benignos asuman cambios cualitativos y se conviertan en un potencial citotóxico destructivo, que a menudo se ve cuando la aparatología fija se ha tenido en los dientes por más de 2 o 3 años. ${ }^{25}$

\section{SELECCION Y PREPARACIÓN DEL PACIENTE}

La ortodoncia acelerada con corticotomías puede realizarse en los casos que estén indicados para un tratamiento de ortodoncia convencional. ${ }^{26}$ Se ha evidenciado que en ortodoncia acelerada con corticotomías ha sido eficiente en el tratamiento de la maloclusión clase I con apiñamiento moderado o severo, la maloclusión clase II que requiere expansión o extracciones, y la maloclusión clase III leve. Para la selección del paciente se debe considerar algunos puntos importantes:

1) Incrementar el efecto del tratamiento de maloclusiones, el límite del movimiento dental y disminuir la necesidad de extracciones.

2) Reducir el tiempo de tratamiento.

3) Desarrollar el volumen alveolar y un periodonto más completo estructuralmente (corrección de dehiscencias y fenestraciones preexistentes).

4) Remodelado alveolar para la mejora sutil del perfil del paciente, cuando está indicado. (La prominencia del mentón sólo puede ser modificada con genioplastias.)

En todos los casos la iniciación de las fuerzas ortodóncicas no debe tardar más de 2 semanas después de la cirugía. Un retraso prolongado dejara de aprovechar al máximo del tiempo del periodo limitado en el que se está dando el RAP. Este periodo es usualmente de 4 a 6 meses, después del cual el movimiento regresa a su velocidad normal. ${ }^{27}$

\section{PROTOCOLOS QUIRÚGICOS}

Se desarrolla en tres fases. La primera es la decorticacion alveolar selectiva (das), se llevan a cabo incisiones sulculares en el alveolo (vestibular y palatino-lingual), formando un colgajo de espesor total, logrando el acceso al hueso alveolar y permitiendo una mejor visualización de la zona, realización de los procesos de aumento y remodelación alveolar (recubrimiento del injerto y mantenimiento de la altura y volumen de los tejidos) y la mejora de la estética gingival donde sea necesario (fig 1 y 2 ).
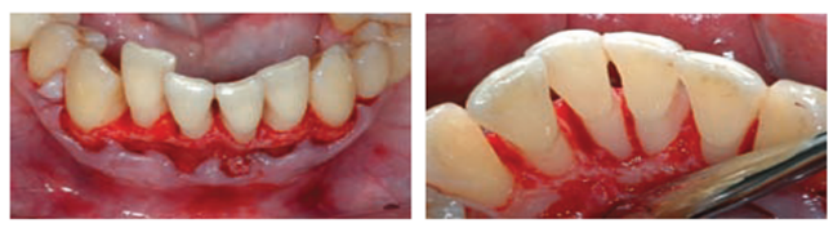

Fig. 1. Elevación del colgajo mucoperiostico a espesor total (vestibular y lingual)

Para las zonas estéticas es recomendable preservar las papilas, el acceso al hueso interproximal se efectúa desde los extremos distales del colgajo haciendo un túnel bajo el mismo. ${ }^{28}$ Posteriormente, se realizan cortes interproximales de $0,5 \mathrm{~mm}$ de profundidad limitados a las corticales vestibular y lingual mediante una fresa redonda para pieza de mano. (fig3) La finalidad de estos cortes es crear una fina capa de hueso $(<1,5 \mathrm{~mm})$ alrededor de la raíz de los dientes y maximizar el sangrado; ${ }^{29}$ es recomendable realizarlos en las paredes más gruesas del hueso cortical, siendo la morfología de los mismos irrelevante (fig. 4) frente al total de la superficie decorticada. $^{30}$

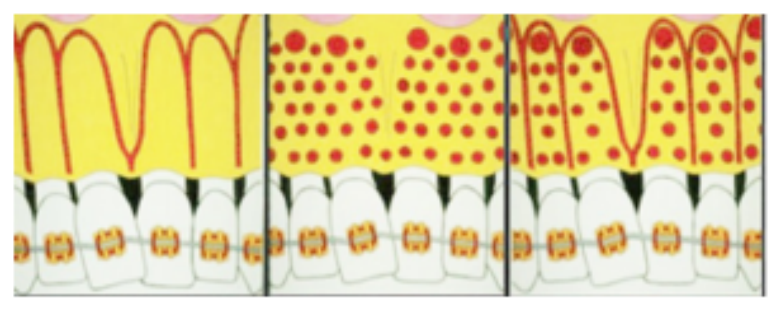

Fig. 2. Combinación de las corticotomías circunscritas con las penetraciones intramedlares.

En la segunda fase se realiza procedimientos de aumento alveolar colocando material reabsorbible en las áreas de decorticacion. Las matrices descalcificadas estimulan la actividad osteoblastica; de esta forma, se obtiene un aumento del volumen alveolar tras el procedimiento. El colgajo se sutura con seda de 4-0, retirándola a las semanas 2-3 si el injerto es superior a $0,5 \mathrm{cc}$. La última fase del procedimiento consiste en la aplicación de las fuerzas ortodonticas, 


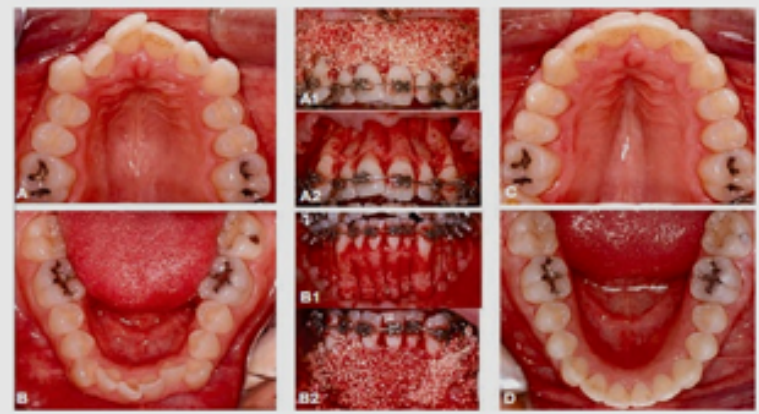

Fig. 3. Corticotomía alveolar selectiva y colocación del injerto oseo.

recomendándose la colocación de la aparatología 1 semana antes de la corticotomia (aunque puede demorarse de 1-2 semanas postcirugia para facilitar el procedimiento). Siendo el ritmo de las activaciones cada 2 semanas. La tasa de movimiento alcanzada con esta técnica es de 1-2mm-semana, frente al milímetro mensual obtenido mediante tratamiento ortodontico convencional. ${ }^{30,31}$

\section{VENTAJAS EVIDENCIADAS}

Reportes de casos clínicos evidencian que esta técnica es segura, efectiva, extremamente predictiva, asociada con la reabsorción radicular y la reducción del tiempo de tratamiento, también puede reducir la necesidad de cirugía ortognática en ciertos casos. Algunas de las ventajas evidenciadas : Tratamientos de mucho menos tiempo a diferencia de los convencionales , ?,23,25,32,33 no altera la vitalidad pulpar o existencia de sensibilidad en piezas dentales tratadas con esta técnica ${ }^{23,24}$,mejora el movimiento dental y reduce la necesidad de extracciones , 7,23,25 evitando la recidiva posterior a la finalización del tratamiento convencional, ${ }^{7}$ menor tiempo en el tratamiento, la flora bacteriana no es afectada por cambios citotóxicos ${ }^{7,25}$, el volumen alveolar aumenta y mejora la estructura del periodonto complejo reduce el tiempo y la carga bacterial, evitando posibles infecciones gracias al injerto óseo que se coloca, el cual previamente fue embebido en una sustancia antibacterial, ${ }^{7}$ no hay formación de bolsas periodontales en las piezas dentarias después del tratamiento ortodóncico con esta técnica,23 disminuye el riesgo de reabsorción radicular. ${ }^{23,25,34,35}$

\section{CONCLUSIONES}

Esta nueva terapia implementada en el tratamiento ortodóntico constituye una herramienta útil en el área de la ortodoncia pues brinda beneficios como una disminución de tiempo de tratamiento, menor reabsorción radicular y una mejor estabilidad una vez el tratamiento haya sido finalizado, se ha evidenciado que la velocidad del movimiento ortodóntico está influenciada por el recambio óseo, la densidad ósea y la hialinización del ligamento periodontal. Esta nueva técnica permite que el especialista brinde una mejor opción en la terapia de sus pacientes, para lograr éxito en esta terapia ortodontica facilitada por corticotomia se requiere cooperación multidisciplinaria entre el ortodoncista y peridoncista.

\section{Referencias}

1 Sebaoun JD, Kantarci A, Turner JW, Carvalho RS, Van Dyke TE, Ferguson DJ. Modeling of trabecular bone and lamina dura following selective alveolar decortication in rats. J Periodontol. 2008;79(9):1679-88.

2 Frost HM. The regional acceleratory phenomenon. A review. Henry Ford Hosp Med J.1983; 31: 3-9.

3 Wilcko MT, Wilcko WM, Bissada NF. An evidence-based analysis of periodontally accelerated orthodontic and osteogénico techniques: A synthesis of scientific perspectives. SeminOrthod. 2008; 14: 305-316.

4 Wilcko MT, Wilcko WM, Breindel-Omniewski K, et al. The periodontally "accelerated osteogenic orthodontics" technique $\left(\mathrm{PAOO}^{\mathrm{TM}}\right)$ technique: Efficient space closing with either orthopedic or orthodontic forces. J Implant AdvClin Dent. 2009; 1: 45- 63.

5 Mavreas D, Athanasiou AE. Factors affecting the duration of orthodontic treatment: a systematic review. Eur J Orthod 2008; 30 (4): 386-95.

6 Wilcko WM, Wilcko T, Bouquot JE, Ferguson DJ. Rapid orthodontics with alveolar reshaping: two case reports of decrowding. Int J PeriodontRestorat Dent 2001; 21 (1): 919.

7 Wilcko MT, Wilcko WM, Bissada NF. An evidence-based analysis of periodontally accelerated orthodontic and osteogenic techniques: a synthesis of scientific perspective. Seminars Orthod 2008; 14: 305-316.

8 Serge Dibart (2007). Practical Advanced Periodontal. Surgery The Contribution of Periodontics to Orthodontic Therapy (pp. 23 ). Editorial Offices: Blackwell Publishing Professional.

9 Köle H. Surgical operations of the alveolar ridge to correct occlusal abnormalities. Oral Surg Oral Med Oral Pathol 1959; 12: 515-529.

10 Henneman S, Von den Hoff JW, Maltha JC. Mechanobiology of tooth movement. Eur J Orthod. 2008;30(3):299-306.

11 Krishnan V, Davidovitch Z. Cellular, molecular, and tissuelevel reactions to orthodontic force. Am J Orthod Dentofacial Orthop 2006; 129 (4): 469e.1-32.

12 Davidovitch Z. Tooth movement. Crit Rev Oral Biol Med 1991; 2 (4): 411-50.

13 Shin MS, Norrdin RW. Regional acceleration of remodeling during healing of bone defect in beagles of various ages. Bone 1985; 6 (5): 377-379.

14 Krishan V, Davidovitch Z. Cellular, molecular, and tissue level reactions to orthodontic force. Am J OrthodDentofacialOrthop. 2006; 129: 469e.1-32.

15 Frost HM. The Biology of fracture healing an overview for clinicians. Part I. ClinOrthopRelat Res 1989; 248: 294-309. 
16 Sebaoun DJ, Kantarci A, Turner JW et al. Modeling of trabecular bone and lamina dura following selective alveolar 31 decortication in rats. J Periodontol 2008; 79 (9): 1679-1688.

17 Lee W, Karapetyan G, Moats R, Yamashita DD, Moon HB, Ferguson DJ, Yen S. Corticotomy-/Osteotomy-assisted tooth movement microCTs differ. J Dent Res 2008; 87 (9): 861-865.

18 Wang L, Lee W, Lei D, Liu Y, Yamashita DD, Yen SLK. Tissue responses in corticotomy and osteotomyassisted tooth movements in grats: histology and immunostaining. Am J OrthodDentofacialOrthop 2009; 136 (6): 770.e1-11.

19 Frost HM. The biology of fracture healing: an overview for 33 clinicians Part I. ClinOrthodont. 1988; 248: 283-293.

20 Yaffe A, Fine N, Binderman I. Regional accelerated phenomenon in the mandible following mucoperiosteal flap surgery. J Periodontol. 1994; 65: 79-83.

21 Wilcko MT, Wilcko W M, Pulver J J, Bissada N F, Bou- 34 quot J E. Accelerated osteogenic orthodontics technique A 1 stage surgically facilitated rapid orthodontic technique with alveolar augmentation. J Oral Maxillofac Surg. 2009; 67:2149-59.

22 Ferguson DJ, Wilcko WM, Wilcko MT. Selective alveolar decortication for rapid surgical-orthodontic resolution of skeletal malocclusion treatment. En Bell WE, Guerrero C. Distraction Osteogenesis of the Facial Skeleton.1ed. Canada: BC Decker; 2007. p. 199-203.

23 Wilcko MW, Wilcko MT, Bouquot JE, Ferguson DJ. Rapid orthodontics with alveolar reshaping: Two case reports of decrowding. Int J PeriodontRestor Dent. 2001; 21(9): 1-11.

24 Lino S, Sakoda S, Ito G, et al. Acceleration of orthodontic tooth movement by alveolar corticotomy in the dog. Am J OrthodDentofacOrthop. 2007; 131: 448.e1-448.e8.

25 Wilcko MT, Wilcko MW, Marquez MG, Ferguson DJ. The contribution of periodontics to orthodontic therapy, En Dibart S. Practical Advanced Periodontal Surgery. 1ed. Ames; Blackwell Munksgaard; 2007. p. 23-50.

26 Murphy KG, Wilcko MT, Wilcko WM, Ferguson DJ. Periodontal accelerated osteogenic orthodontics: a description of the surgical technique. J Oral Maxillofac Surg. 2009; 67(10): 2160-6.

27 AlGhamdi AST. Corticotomy facilitated orthodontics: Review of a technique. Saudi Dental J. 2010; 22(1): 1-5.

28 Murphy KG, Wilcko MT, Wilcko WM, Fergusn DJ. Periodontal accelerated osteogenic orthodontics: a description of the surgical technique. J Oral Maxillofac Surg. 2009:67(10):2160-6.

29 Wilcko MT, Wilcko WM, Pulver JJ, Bissada NF, Bouquot JE. Accelerated osteogenic orthodontics technique: a I-estage surgically facilitated rapid orthodontics technique with alveolar augmentation. J Oral maxillofac Surg. 2009;67(10):2149-59.

30 Davidvotich Z, Murphy N. The adaptation and development of biological concept in orthodontics. In: Krishnan V, Davidovtich $\mathrm{Z}$. Biological mechanisms of tooth movement.
Wiley-Blackwell; 2009, p, 8-13.

Murphy NC. In vivo tissue engineering for orthodontics: a model frist step. In: Davidovitch Z, Mah J, Suthanarak S, eds. Biological mechanisms of tooth eruption, resorption and movement. Boston, MA: The Harvard Society for the Advancement of Orthodontics; 2006. P. 385-410.

32 Ferguson DJ, Wilcko WM, Wilko MT. Selective alveolar decortication for rapid surgical-orthodontic of skeletal malolusion treatment. In:Bell WE. Guerrero C. Distraction osteogenesis of the facial skeleton. Hamilton, ON:BC Decker, Inc; 2007. P. 199-203.

Bolding SL, Roblee RR, Sándor G, Charles M. Optimizing orthodontic therapy with dentoalveolar distraction osteogenesis. En Bell WE, Guerrero C. Distraction Osteogenesis of the Facial Skeleton. 1 ed. Canada: BC Decker; 2007. p. 187197.

4 Wang L, Lee W, Lei DL, Liu YP, Yamashita DD, Yen SL. Tisssue responses in corticotomy- and osteotomy-assisted tooth movements in rats: histology and immunostaining. Am J Orthod Dentofacial Orthop. 2009;136(6):770.e1-11.

35 Lino S, Sakoda S, Ito G, et al. Acceleration of orthodontic tooth movement by alveolar corticotomy in the dog. Am J Orthod Dentofac Orthop. 2007; 131:448.e1-448.8.

Recibido: 16 de Junio de 2017.

Aceptado: 20 de Octubre de 2017. 
